\title{
IDENTIFICACIÓN DE MICOBACTERIAS EN MEDIO SÓLIDO MEDIANTE MICROSCOPÍA DE FASE INVERTIDA Y TINCIÓN ZIEHL-NEELSEN
}

\author{
Jesús Rojas Jaimes ${ }^{1,2, a}$, Jorge Giraldo-Chavez ${ }^{3, b}$, Yudit Huyhua-Flores ${ }^{3, c}$, Tatiana Caceres-Nakiche ${ }^{3, b}$
}

\begin{abstract}
RESUMEN
Con el objetivo de identificar diferencias morfológicas en colonias de diferentes especies de micobacterias mediante microscopía de fase invertida, se sembraron en medio sólido $7 \mathrm{H} 11$, nueve especies de micobacterias patógenas de alta prevalencia y se observaron hasta por 21 días. Adicionalmente se realizaron coloraciones Ziehl-Neelsen (ZN) para cada una de ellas. No se identificó variaciones morfológicas entre las especies cultivadas, sin embargo, se observó que todas fueron capaces de formar cordones en su etapa temprana de crecimiento, siendo corroborado por coloración ZN en Mycobacterium tuberculosis. Asimismo, se determinó ciertas características microbiológicas como tiempo mínimo y la temperatura de crecimiento, y características bacilares basadas en la tinción ZN para cada especie estudiada. Estos hallazgos son importantes para la identificación del agente etiológico y podrían servir de base para estudios posteriores sobre la identificación de las biomoléculas involucradas en la agregación celular de cada micobacteria.
\end{abstract}

Palabras claves: Tuberculosis; Mycobacterium tuberculosis; Micobacterias; Medios de cultivo (fuente: DeCS BIREME).

\section{MYCOBACTERIA IDENTIFICATION IN SOLID CULTURE BY INVERTED PHASE MICROSCOPY AND ZIEHL-NEELSEN STAIN}

\begin{abstract}
With the aim of identifying morphological differences in colonies of different species of mycobacteria by means of inverted phase microscopy, nine species of high-prevalence pathogenic mycobacteria were seeded in $7 \mathrm{H} 11$ solid medium and were observed for up to 21 days. Additionally, Ziehl-Neelsen (ZN) stains were carried out for each one of them. No morphological variations were identified among the species grown on solid medium; however, it was identified that all are able to form cords in solid medium in an early stage of growth, being corroborated by the Ziehl-Neelsen stain in Mycobacterium tuberculosis. so techniques based on detecting $M$. tuberculosis depending on the identification of the cords should be taken with caution so as not to generate a bad diagnosis, so microbiological characteristics could also be established as a minimum time to detect growth, temperature of growth and bacillary characteristics based on the Ziehl-Neelsen stain for each species studied.
\end{abstract}

Keywords: Tuberculosis; Mycobacterium tuberculosis; Mycobacterias; Culture media (source: MeSH NLM).

\section{INTRODUCCIÓN}

Latuberculosis (TB) es una de las enfermedades ampliamente distribuidas y más devastadoras en todo el mundo debido a su alta prevalencia, morbilidad y mortalidad, además de su facilidad de propagación ${ }^{(1)}$.

Métodos moleculares disponibles en el mercado para la identificación del agente etiológico de la TB representan una herramienta útil y prometedora, pero son caros y no cubren la variedad de micobacterias que pueden ser identificadas con buena fiabilidad en los laboratorios clínicos ${ }^{(2)}$.

La tipificación del agente etiológico es importante debido a que las infecciones producidas por diferentes especies de micobacterias pueden requerir un tratamiento diferente (3). Algunas de las técnicas de identificación son demasiado

\footnotetext{
Universidad Científica del Sur, Escuela de Medicina Humana. Lima, Perú

Universidad Continental, Facultad de Ciencias Básicas. Lima, Perú

Universidad Peruana Cayetano Heredia, Instituto de Enfermedades Tropicales Alexander von Humboldt. Lima, Perú

Magister en Biología Molecular; ${ }^{b}$ biólogo; ${ }^{c}$ técnico de laboratorio

Recibido: 13/02/2018 Aprobado: 16/05/2018 En línea: 05/06/2018
}

Citar como: Rojas Jaimes J, Giraldo-Chavez J, Huyhua-Flores Y, Caceres-Nakiche T. Identificación de micobacterias en medio solido mediante microscopía de fase invertida y tinción Ziehl-Neelsen. Rev Peru Med Exp Salud Publica. 2018;35(2):279-84. doi:10.17843/rpmesp.2018.352.3471. 
complejas y/o de costo no accesible para muchos centros hospitalarios ${ }^{(4,5)}$. El diagnóstico de TB activa se basa principalmente en la constatación directa de los bacilos, ya sea en frotis de esputo o en cultivo.

Los procedimientos que son operador-dependiente como la baciloscopia no son lo suficientemente sensibles para detectar más del 65 - $70 \%$ de la carga bacteriana en la enfermedad ${ }^{(6)}$. La prueba Microscopic-Observation DrugSusceptibility (MODS) desarrollada en el Perú se basa en un medio líquido en placas de cultivo de tejidos de 24 pocillos para la detección temprana de crecimiento, mediante la observación por microscopía de fase invertida para la detección de la formación típica del cordón de Mycobacterium tuberculosis ( $M$. tuberculosis), con una mayor sensibilidad que otros cultivos clásicos como el automatizado o el Löwenstein-Jensen ${ }^{(7)}$. Sin embargo, este método no puede diferenciar entre especies de micobacterias, necesitándose una combinación de técnicas ${ }^{(7,8)}$.

Existen componentes bacterianos que podrían indicar diferencias, como los ácidos grasos ${ }^{(9)}$ y la micosida, un componente bacteriano que está vinculado a la forma de la colonia y a la fisiología de Mycobacterium kansasii y Mycobacterium fortuitum ${ }^{(10)}$. Estudios previos muestran la importancia de los lípidos, glicopeptolípidos y proteínas en la fisiología y disposición de la pared bacteriana de Mycobacterium smegmatis y Mycobacterium avium, quienes vinculan estos componentes a la forma de sus colonias, resistencia y virulencia, incluso los lípidos de su pared bacteriana son especie-específicos ${ }^{(11-13)}$.

Mutaciones en los genes de la especie Mycobacterium abscessus están involucrados en la síntesis de la trehalosa 6,6- dimicolato relacionado con la formación de cordones, los cuales se reconocen como factores de virulencia y producen cambios morfológicos en la colonia ${ }^{(14)}$. En Mycobacterium marinum se ha reportado que las múltiples mutaciones en las proteínas ESAT-6, EsxB producen cambios estructurales en la forma de la colonia ${ }^{(15)}$.

La identificación de micobacterias consume mucho tiempo en los medios sólidos tradicionales y deben ser complementadas por técnicas moleculares que son caras, además de necesitar un personal altamente entrenado.

Por lo tanto, es necesario encontrar un método de identificación que sea rápido, seguro, barato y de menor complejidad, comparado a los métodos convencionales. Por tal motivo el objetivo de nuestro estudio fue identificar diferencias morfológicas en colonias de diferentes especies de micobacterias mediante microscopía de fase invertida en medio sólido $7 \mathrm{H} 11$ y tinción Ziehl-Neelsen.

\section{MENSAJES CLAVE}

Motivación para realizar el estudio. La identificación de las especies de micobacterias es costosa, compleja por el nivel de laboratorios requeridos y tarda tiempo.

Principales hallazgos. Las micobacterias en el estudio formaron cordones en medio sólidos. La coloración Ziehl Neelsen (ZN) mostro diferentes patrones de agregación celular desde los unicelulares, cadenas y cordones en diferentes micobacterias.

Implicancias. Se debe de tener en cuenta el cuidado en la caracterización de las micobacterias basada solo en la formación de cordones, debiendo complementarse con la coloración ZN. El medio solido 7H11 es de mucha utilidad en la detección temprana del bacilo mediante microscopía.

\section{EL ESTUDIO}

\section{DISEÑO Y POBLACIÓN}

Se realizó una investigación descriptiva microbiológica basado en la diferenciación colonial y celular de diferentes especies de micobacterias patógenas. Las especies de micobacterias fueron obtenidas del Instituto de Medicina Tropical de la Universidad Peruana Cayetano Heredia (UPCH) y del College of American Pathologists (CAP). Se utilizaron las siguientes especies: Mycobacterium gordonae (CAP), mycobacterium avium (secuenciamiento), Mycobacterium marinum (UPCH), Mycobacterium smegmatis (UPCH), Mycobacterium fortuitum (CAP), Mycobacterium chelonae (CAP), Mycobacterium terrea (UPCH), mycobacterium neonarum (CAP) y M. tuberculosis (Cepa H37Rv ATCC).

Las especies utilizadas estuvieron preservadas en medio Indicador de Crecimiento de Micobacteria en Tubo (MIGT). Se extrajo una alícuota del medio MIGT para cada especie y se ajustó en un tubo estéril a escala 5 de McFarland. Posteriormente se realizó una dilución $1 / 10$ para ser sembradas en placas en medio sólido $7 \mathrm{H} 11$.

\section{PREPARACIÓN DE MEDIO SÓLIDO 7H11 Y SIEMBRA}

El medio $7 \mathrm{H} 11$ fue preparado según indicaciones del proveedor, se adicionó $100 \mathrm{ml}$ de una mezcla de ácido oleico, albúmina, dextrosa y catalasa (OADC), mezclando por cinco minutos ${ }^{(16)}$. Se añadió $25 \mathrm{ml}$ de ácido paranitro benzoico (PNB) y se repitió la mezcla por cinco minutos. Se dispensó cuatro $\mathrm{ml}$ aproximadamente hasta llenar la placa de 90 x 15 mm y finalmente se dejó gelificar sin exposición a la luz, empaquetando las placas en papel Kraft hasta su uso. Se realizó el control de esterilidad a $37^{\circ} \mathrm{C}$ por 24 horas y luego se guardó en refrigeración entre cuatro a ocho ${ }^{\circ} \mathrm{C}$. Las placas fueron utilizadas hasta un máximo de 30 días pospreparación. 
Se extrajo $200 \mu \mathrm{l}$ de la dilución 1/10, con una pipeta de un $\mathrm{ml}$ y se colocó en cinco puntos equidistantes en la placa de $7 \mathrm{H} 11$, se homogenizó manualmente moviendo la placa en círculos concéntricos para que la siembra se extienda. Cada especie de micobacteria fue sembrada en tres placas para disponer de dos repeticiones por especie.

\section{LECTURA E INTERPRETACIÓN}

Las placas sembradas de medio $7 \mathrm{H} 11$ se expusieron entre $32^{\circ} \mathrm{C}$ y $37^{\circ} \mathrm{C}$, dependiendo de la especie de micobacteria utilizada. Fueron colocadas en una incubadora $\sin \mathrm{CO}_{2}$ en un laboratorio nivel III de la Universidad Peruana Cayetano Heredia. La lectura se realizó cada día excepto los sábados y domingos por un máximo de 21 días, usando un microscopio de fase invertida con aumento total de 100X. Al identificar el crecimiento microscópico se procedió a la toma fotográfica en cada muestra identificada y se realizó el seguimiento microscópico diario hasta que las colonias presenten una forma ovoide. Se evaluó la forma típica de cada colonia y la formación de cordones en cada especie de micobacteria estudiada.

\section{TINCIÓN ZIEHL NEELSEN (ZN)}

Del tubo 5 de McFarland se colocó una alícuota de $50 \mu \mathrm{l}$, se extendió en una lámina portaobjeto limpia. Luego de su secado, se agregó gotas de fucsina fenicada hasta cubrir las muestras, se realizó el calentamiento de la muestra por cinco minutos y se decoloró con alcohol ácido, se adicionó el colorante de contraste azul de metileno por un minuto, luego se lavó y seco para ser leída con un aumento de 1000X. Para cada especie de micobacteria el procedimiento de tinción se realizó por triplicado. Se evaluó la presencia de bacterias alcohol ácido resistente, así como la formación de cordones (agregación multicelular con disposición de cordones) y formación de cadenas (agregación unicelular en continuidad).

\section{RESULTADOS}

La primera identificación microscópica de las colonias de micobacterias se realizó entre el primer y decimocuarto día (Tabla 1). Todas las micobacterias cultivadas en el medio sólido $7 \mathrm{H} 11$ presentaron el mismo patrón de crecimiento colonial con presencia de cordones claramente visibles, no se encontró diferencias morfológicas entre las diferentes especies de micobacterias estudiadas (Figuras 1 y 2 ).

Respecto a la identificación celular de las micobacterias por ZN, se encontraron patrones unicelulares, cadenas y cordones (Figuras 3, 4 y 5).

Todas las especies fueron cultivadas y teñidas por $\mathrm{ZN}$ con dos repeticiones, mostrando el mismo patrón microbiológico para cada especie.

Tabla 1. Características microbiológicas de las micobacterias en medio 7H11 a nivel microscópico

\begin{tabular}{|c|c|c|c|c|}
\hline Especie de micobacteria & $\begin{array}{c}\text { Tiempo mínimo } \\
\text { para la detección } \\
\text { microscópica } \\
\text { (días) }\end{array}$ & $\begin{array}{l}\text { Temperatura de } \\
\text { crecimiento } \\
\text { (Grados } \\
\text { Celsius }-{ }^{\circ} \mathrm{C} \text { ) }\end{array}$ & $\begin{array}{c}\text { Características } \\
\text { macroscópicas de la } \\
\text { colonia }\end{array}$ & $\begin{array}{c}\text { Características de lectura } \\
\text { Ziehl-Neelsen }\end{array}$ \\
\hline M. fortuitum & 3 & 37 & $\begin{array}{l}\text { Marfil, bordes } \\
\text { irregulares y lisa }\end{array}$ & $\begin{array}{l}\text { Formación de } \\
\text { cadenas cortas }\end{array}$ \\
\hline M. chelonae & 3 & 32 & $\begin{array}{l}\text { Blanca, bordes } \\
\text { irregulares y lisa }\end{array}$ & $\begin{array}{l}\text { Bacilos unicelulares y/o } \\
\text { cadenas cortas }\end{array}$ \\
\hline M. smegmatis & 9 & 32 & $\begin{array}{l}\text { Crema, bordes } \\
\text { irregulares y lisa }\end{array}$ & $\begin{array}{l}\text { Formación de } \\
\text { cadenas cortas }\end{array}$ \\
\hline M. marinum & 1 & 32 & $\begin{array}{l}\text { No detectado hasta } \\
\text { los } 21 \text { días }\end{array}$ & $\begin{array}{l}\text { Bacilos unicelulares } \\
\text { cortos }\end{array}$ \\
\hline M. gordonae & 1 & 37 & $\begin{array}{l}\text { No detectado hasta } \\
\text { los } 21 \text { días. }\end{array}$ & $\begin{array}{l}\text { Formación de } \\
\text { cadenas largas }\end{array}$ \\
\hline M. terrae & 1 & 37 & $\begin{array}{l}\text { No detectado hasta } \\
\text { los } 21 \text { días. }\end{array}$ & $\begin{array}{l}\text { Bacilos unicelulares } \\
\text { pequeños y cortos }\end{array}$ \\
\hline M. neoaurum & 1 & 37 & $\begin{array}{l}\text { Amarilla, bordes } \\
\text { regulares y lisa }\end{array}$ & $\begin{array}{c}\text { Bacilos unicelulares cortos y } \\
\text { gruesos }\end{array}$ \\
\hline M. avium & 3 & 37 & $\begin{array}{l}\text { Marfil, bordes } \\
\text { irregulares y lisa }\end{array}$ & Diplobacilos \\
\hline M. tuberculosis & 14 & 37 & $\begin{array}{l}\text { Blanca, bordes } \\
\text { irregulares y lisa }\end{array}$ & $\begin{array}{l}\text { Formación de } \\
\text { cordones }\end{array}$ \\
\hline
\end{tabular}




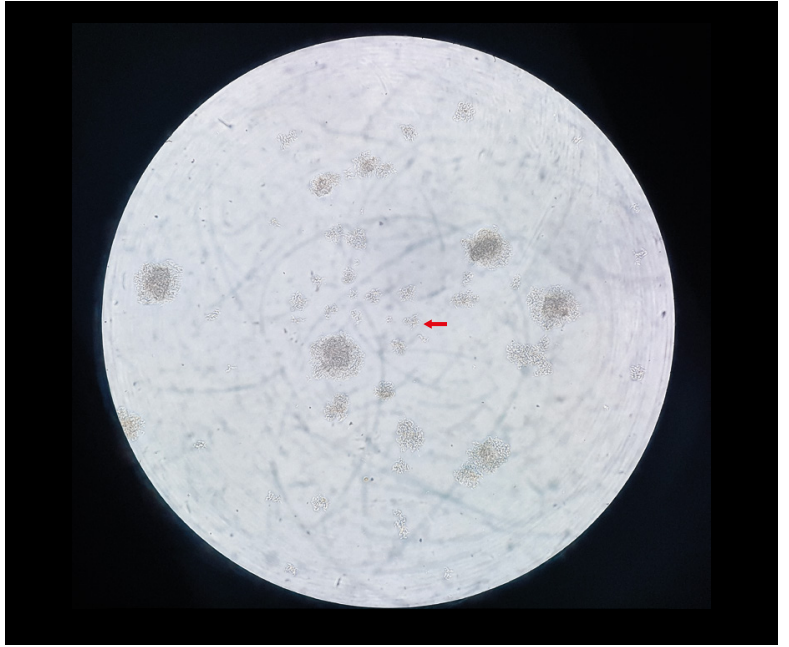

Figura 1. Microfotografía a los 14 días de crecimiento de $M$. tuberculosis, 100X. La misma formación colonial se observó en todas especies de micobacterias estudiadas, así como en sus repeticiones de los cultivos correspondientes

\section{DISCUSIÓN}

Se ha realizado una investigación bacteriológica en diferentes especies de micobacterias patógenas encontradas frecuentemente en el aislamiento de muestras clínicas ${ }^{(17,18)}$. En las nueve especies de micobacterias estudiadas no hallamos diferencias significativas respecto a la morfología de las colonias en medio sólido $7 \mathrm{H} 11$ por microscopía de fase invertida, a pesar de que estudios previos hacen presumir la diferenciación colonial microscópica de diferentes especies, fundamentada en las moléculas lipídicas, proteicas y azúcares ${ }^{(9,11-13)}$.

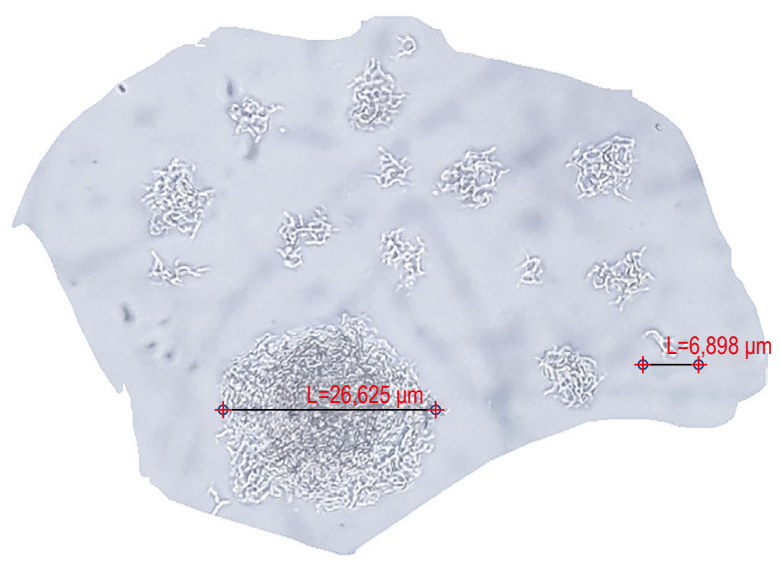

Figura 2. Microfotografía a los 14 días de crecimiento, se observan cordones de $M$. tuberculosis, 100X. Los cordones se pudieron observar en todas las micobacterias en estudio (flecha roja)

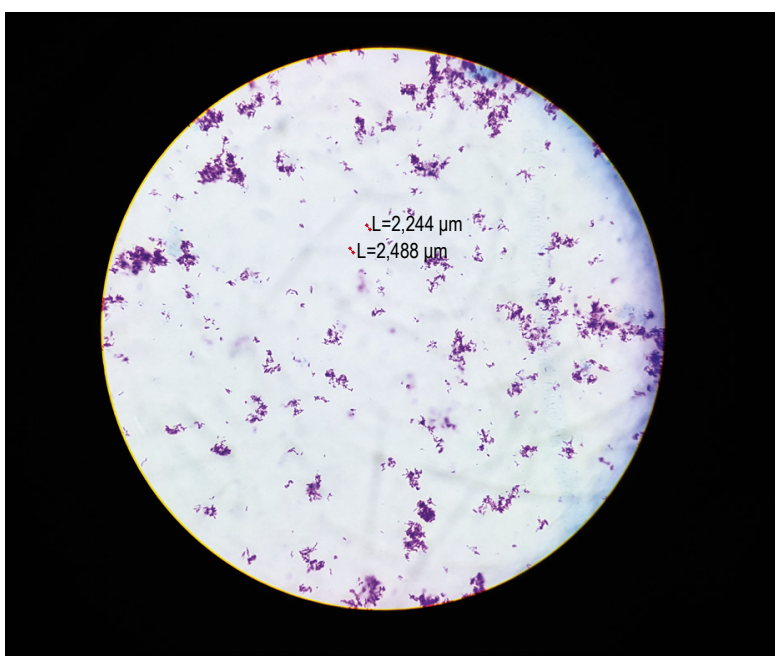

Figura 3. Lectura Ziehl Neelsen de M. marinum, se observan bacilos unicelulares a $1000 \mathrm{X}$

Sin embargo, pudimos encontrar diferencias respecto al tiempo mínimo para la detección microscópica, este varió entre un día y 14 días siendo un resultado importante para la determinación temprana de una micobacteria. Un hallazgo de suma importancia fue la formación de cordones, a nivel microscópico, en todas las especies evaluadas en medio $7 \mathrm{H} 11$, a pesar que la literatura limita la característica de formación de cordones principalmente a $M$. tuberculosis. Asimismo, en esta especie también se identificó cordones en muestras teñidas con coloración $\mathrm{ZN}$, siendo la única con esta característica, tanto en el medio líquido MIGT como en el medio sólido $7 \mathrm{H} 11$.

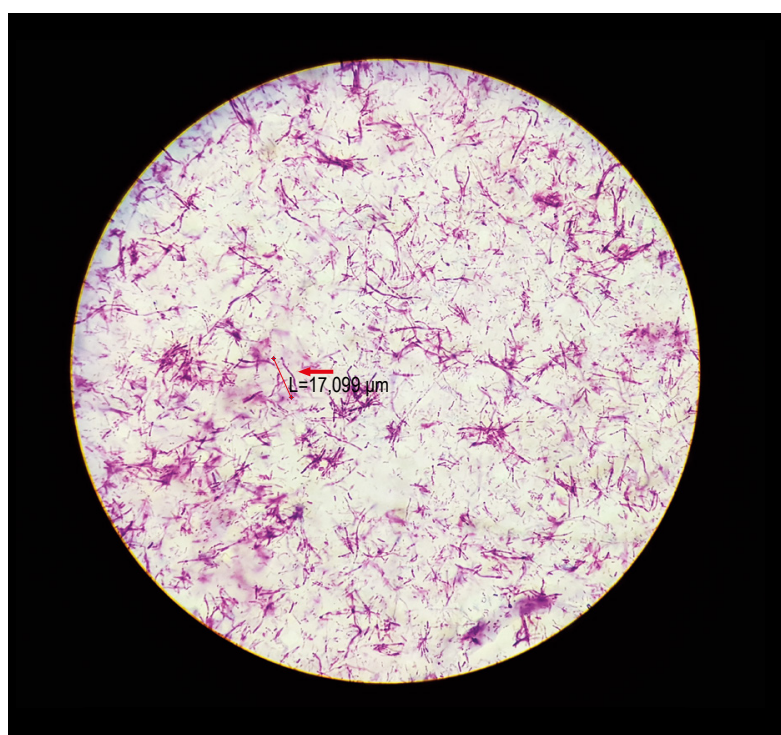

Figura 4. Lectura Ziehl Neelsen de M. gordonae, se observan cadenas a $1000 \mathrm{X}$ 


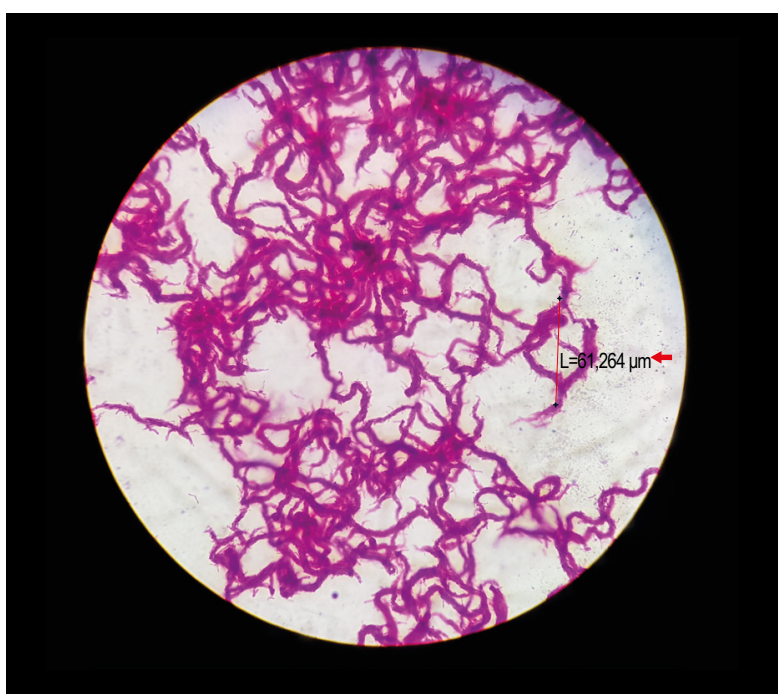

Figura 5. Lectura Ziehl Neelsen de M. tuberculosis, se observan cordones a $1000 \mathrm{X}$

Las tendencias a la agrupación celular se observaron en diferentes grados en las muestras teñidas con el método de ZN para cada especie de micobacteria, por ejemplo, Mycobacterium chelonae, Mycobacterium marinum, Mycobacterium terrae y Mycobacterium neoaurum mostraron un patrón de bacilos no agrupados; en Mycobacterium avium formaban diplobacilos; en Mycobacterium fortuitum y Mycobacterium smegmatis formaban cadenas cortas y en Mycobacterium gordonae se observó cadenas largas. En M. tuberculosis se observó la formación de cordones con el mayor patrón de agrupación, esto podría relacionarse con la virulencia de la bacteria según lo mencionado en estudios previos ${ }^{(14,18)}$.

Las diferentes especies de micobacterias estudiadas son capaces de formar cordones en etapas tempranas en el medio $7 \mathrm{H} 11$, tal como se observan en las figuras. La formación de cordones fue reportada por primera vez por Robert Koch y fue atribuida a una relación entre la virulencia y el fenotipo de $M$. tuberculosis ${ }^{(18)}$, posterior a esto, otros estudios reportaron la formación de cordones por otras especies de micobacterias ${ }^{(19)}$.

La relevancia clínica de estos hallazgos radica en que varias técnicas de diagnóstico como el MODS se basan en la identificación microscópica de los cordones en $M$. tuberculosis ${ }^{(16)}$, esto podría conducira una mala identificación de otras especies de micobacterias, que también presentan cordones en medio sólido, ocasionando un mal diagnóstico. Incluso se podría reportar una $M$. tuberculosis resistente a las drogas cuando en realidad es otra micobacteria con resistencia natural a ciertas drogas antituberculosas, siendo necesario otro esquema terapéutico ${ }^{\left({ }^{34}\right)}$. Adicionalmente, sería importante tomar en cuenta al medio sólido $7 \mathrm{H} 11$ para el aislamiento e identificación de resistencia a drogas debido a la mejor bioseguridad y al corto tiempo de crecimiento de las micobacterias, comparado con el MODS o el medio de Löwenstein-Jensen.

Las limitaciones del presente estudio fueron: no realizar microscopía electrónica para observar la ultraestructura de agregación a nivel celular de las micobacterias; no incluir al $M$. tuberculosis resistente para observar sus características morfológicas a nivel microscópico, y no identificar los componentes moleculares de las especies que están relacionadas con la estructura colonial. A pesar de las limitaciones el presente trabajo es innovador y resalta los hallazgos de la identificación de cordones en todas las micobacterias estudiadas, pudiéndose observar la agregación y la formación de cordones, incluso desde etapas muy tempranas, como se comprobó en la tinción ZN de M. tuberculosis. Estos hallazgos son de suma importancia para el correcto diagnóstico del agente etiológico, así como el uso de la microscopía de fase invertida y el medio sólido $7 \mathrm{H} 11$.

Se concluye, que todas las especies de micobacterias estudiadas fueron capaces de formar cordones en fase temprana, en medio $7 \mathrm{H} 11$, usando microscopía de fase invertida. Utilizando la coloración ZN se encontraron patrones diferenciales en la agregación celular en la mayoría de las especies, siendo estos hallazgos de suma importancia para la identificación del agente etiológico. Estos hallazgos podrían servir de base para estudios posteriores sobre la identificación de las biomoléculas involucradas en la agregación celular, relacionada con la virulencia de cada micobacteria.

Agradecimientos: Al Dr. Carlton Evans del Imperial College London por el apoyo logístico al presente proyecto y la Universidad Científica del Sur por el apoyo con el financiamiento al presente proyecto.

Contribuciones de autoría: JRJ participó en la concepción y diseño del artículo; análisis e interpretación de datos; redacción del artículo; revisión crítica del artículo y aprobación de la versión final. JGC: participó en la recolección de resultados; análisis e interpretación de datos. YHF: participó en la recolección de resultados y análisis e interpretación de datos. TCN participó en la asesoría técnica o administrativa y aporte de pacientes o material de estudio.

Fuente de financiamiento: El presente trabajo fue financiado por la Universidad Científica del Sur a través de su área de investigación.

Conflictos de interés: Los autores declaran no tener conflictos de interés. 


\section{REFERENCIAS BIBLIOGRÁFICAS}

1. Palomino JC, Leao SC, Ritacco V. Tuberculosis 2007 From basic science to patient care. lera edición [Internet], São Paulo: TuberculosisTextbook.com; 2007. Disponible en: http://pdfflyingpublisher. com/tuberculosis2007.pdf

2. dos Santos RP, Scheid KL, Willers DM, Goldani LZ. Comparative radiological features of disseminated disease due to Mycobacterium tuberculosis nontuberculosis mycobacteria among AIDS patients in Brazil. BMC Infect Dis. 2008;8:24. doi: 10.1186/1471-2334-8-24.

3. Kremer $K$, van-der-Werf MJ, Au BK, Anh DD, Kam KM, van-Doorn HR, et al. Vaccine-induced Immunity Circumvented by typical Mycobacterium tuberculosis Beijing Strains. Emerg Infect Dis. 2009;15(2):335-9.

4. Shiferaw G, Woldeamanuel Y, Gebeyehu M, Girmachew F, Demessie D, Lemma E. Evaluation of Evaluation of microscopic observation drug susceptibility assay for detection of multidrug-resistant Mycobacterium tuberculosis. J Clin Microbiol. 2007;45(4):1093-7.

5. Moore DA, Evans CA, Gilman RH, Caviedes L, Coronel J, Vivar A, et al. Microscopic-Observation DrugSusceptibility Assay for the Diagnosis of TB. N Engl J Med. 2006;355(15):1539-50.

6. Kashino SS, Pollock N, Napolitano DR, Rodrigues V Jr, Campos-Neto A. Identification and Characterization of Mycobacterium tuberculosis antigens in urine of patients with active pulmonary tuberculosis:an innovative and alternative approach of antigen Discovery of useful microbiol molecules. Clin Exp Immunol.
2008;153(1):56-62. doi: 10.1111/j.1365 2249.2008.03672.x.

7. Ernst JD, Trevejo-Nuñez G, Banaiee N. Genomics and the evolution,patogénesis ,and diagnosis of Tuberculosis. J Clin Invest. 2007;117(7):1738-45.

8. Breen RA, Hardy GA, Perrin FM, Lear S, Kinloch S, Smith CJ, et al. Rapid Diagnosis of Smear Negative Tuberculosis Using Immunology and Microbiology with Induced Sputum in HIV-infected and uninfected individual. PLoS One. 2007;2(12):e1335.

9.- Selvarangan R, Wu WK, Nguyen TT, Carlson LD, Wallis CK, Stiglich SK, et.al. Characterization of a Novel Group of Mycobacteria and Proposal of Mycobacterium sherrisii sp. nov. J Clin Microbiol. 2004;42(1):52-9.

10. Fregnan GB, Smith DW, Randall HM. Biological and Chemical Studies on mycobacteria relationship of colony morphology to mycoside content for Mycobacterium kansasii and Mycobacterium fortuitum.J Bacteriol. 1961;82:517-27.

11.- Chen JM, German GJ, Alexander DC, Ren H, Tan T, Liu J. Roles of Lsr2 in Colony Morphology and Biofilm Formation of Mycobacterium smegmatis. J Bacteriol. 2006;188(2):633-41.

12. Recht J, Kolter R. Glycopeptidolipid Acetylation Affects Sliding Motility and Biofilm Formation in Mycobacterium smegmatis. J Bacteriol. 2001;183(19):571824.

13. Fregnan GB, Smith DW, Randall HM. A Mutant of a Scotochromogenic Mycobacterium detected by colony morphology and lipid studies. J Bacteriol. 1962;83:828-36.
14.-Byrd T, Lyons R. Preliminary Characterization of a Mycobacterium abscessus Mutant in Human and Murine Models of Infection. Infect Immun. 1999;67(9):4700-7.

15. Kennedy GM, Hooley GC, Champion MM, Mba Medie F, Champion PA. A Novel ESX-1 Locus Reveals that Surface-Associated ESX-1 Substrates Mediate Virulence in Mycobacterium marinum. J Bacteriol. 2014May;196(10):1877-88. doi: 10.1128/ JB.01502-14.

16. MIDDLEBROOK 7H11 AGAR (THIN POUR) [Internet]. Kansas: Thermo Fisher Scientific; 2011. Disponible en: https://assets.thermofisher.com/TFS-Assets/LSG/manuals/IFU1606-PI.pdf

17. Dorronsoro I, Torroba L. Microbiología de la tuberculosis. An Sist Sanit Navar. 2007;30(2): 67-84.

18. Kim CK, Choi SI, Jeon BR, Lee YW, Lee YK, Shin HB. Pulmonary Infection Caused by Mycobacterium neoaurum: The First Case in Korea. Ann Lab Med. 2014;34(3):243-6. doi: 10.3343/ alm.2014.34.3.243.

19. Sánchez-Chardi A, Olivares F, Byrd T, Julián E, Brambilla E, Luquin M. Demonstration of Cord Formation by Rough Mycobacterium abscessus Variants: Implications for the Clinical Microbiology Laboratory. J Clin Microbiol. 2011 Jun;49(6):2293-5. doi: 10.1128/JCM.02322-10.

Correspondencia: Jesús Rojas Jaimes

Dirección: Panamericana Sur 19, Villa EL

Salvador 15067. Lima, Perú

Teléfono: (511) 993638840

Correo electrónico:jesus.rojas.jaimes@gmail.com 